\title{
VIM3 Corrigendum available online
}

A Corrigendum to the "International vocabulary of metrology_Basic and general concepts and associated terms (VIM)" became available in May 2010. A PDF version of this list of corrections of typographic and editorial imperfections can be downloaded free of charge from the BIPM website, i.e. from the same site as VIM3 itself, at http://www.bipm.org/en/publications/guides/vim.

Please note that the Corrigendum does not include any changes of content. In the daily work in chemical or clinical laboratories, the corrections indicated may be relevant in particular cases only. However, they are well worth to be noted in view of the paramount importance of VIM3, which meets the conceptual needs of measurement throughout physics, chemistry, laboratory medicine, biology, and engineering, on the basis that there is no fundamental difference in the basic principles of measurement.

The publication of VIM3 online in 2008 answered fundamental questions about current philosophies and descriptions of measurement. It marked a change of paradigm by replacing the Error Approach with an Uncertainty Approach, which had been detailed in the "Guide to the expression of uncertainty in measurement" (http://www. bipm.org/en/publications/guides/gum including the 2008 Supplement). Application of this approach in the practice of chemical measurements and its further development are still favorite topics highlighted in ACQUAL's articles.

The Corrigendum was prepared by the Working Group 2 of the Joint Committee for Guides in Metrology (JCGM) and released by the JCGM whose member societies had jointly initiated, and subsequently approved VIM3 and share the copyright. These societies are the International Bureau of Weights and Measures (BIPM), the International Electrotechnical Commission (IEC), the International Federation of Clinical Chemistry and Laboratory Medicine (IFCC), the International Laboratory Accreditation Cooperation (ILAC), the International Organization for Standardization (ISO), the International Union of Pure and Applied Chemistry (IUPAC), the International Union of Pure and Applied Physics (IUPAP), and the International Organization of Legal Metrology (OIML).

(Source: http://www.bipm.org/en/publications/guides/vim). 